\title{
LA COMPENSATION ÉCOLOGIQUE : MARCHÉ OU MARCHANDAGE ?
}

Valérie Boisvert

De Boeck Supérieur | «Revue internationale de droit économique »

2015/2 t. XXIX | pages 183 à 209

ISSN 1010-8831

ISBN 9782807301177

Article disponible en ligne à l'adresse :

http://www.cairn.info/revue-internationale-de-droit-economique-2015-2-page-183.htm

\section{!Pour citer cet article :}

Valérie Boisvert, «La compensation écologique : marché ou marchandage ? », Revue internationale de droit économique 2015/2 (t. XXIX), p. 183-209.

DOI 10.3917/ride.292.0183

Distribution électronique Cairn.info pour De Boeck Supérieur.

(C) De Boeck Supérieur. Tous droits réservés pour tous pays.

La reproduction ou représentation de cet article, notamment par photocopie, n'est autorisée que dans les limites des conditions générales d'utilisation du site ou, le cas échéant, des conditions générales de la licence souscrite par votre établissement. Toute autre reproduction ou représentation, en tout ou partie, sous quelque forme et de quelque manière que ce soit, est interdite sauf accord préalable et écrit de l'éditeur, en dehors des cas prévus par la législation en vigueur en France. Il est précisé que son stockage dans une base de données est également interdit. 


\title{
LA COMPENSATION ÉCOLOGIQUE : MARCHÉ OU MARCHANDAGE ?
}

\author{
Valérie BOISVERT ${ }^{1}$
}

Résumé : Les « banques de conservation 》 sont fréquemment présentées comme de nouveaux instruments de marché au service de la conservation de la biodiversité. Il s'agit d'une modalité de mise en ouvre de la compensation écologique d'abord développée aux États-Unis au début des années 2000, avant d'être plus globalement diffusée. Ce dispositif est perçu par ses promoteurs comme un marché en devenir, tandis que ses détracteurs y voient le dernier avatar du projet de marchandisation de la nature au cour des politiques environnementales depuis deux décennies. Audelà des discours, les arrangements institutionnels en jeu dans les banques de conservation sont mal connus. Cet article se propose de revenir sur leur statut économique en analysant le dispositif tel que décrit dans la réglementation fédérale qui lui est consacrée aux États-Unis et en évaluant sa mise en place à l'aune de critères et attributs constitutifs d'un marché, qui auront été préalablement définis.

1 Introduction

2 La nature des marchés de la nature

2.1 Le développement des marchés et des instruments de marché : quelle légitimité théorique?

2.2 La construction des marchandises et des arrangements marchands

3 Les banques de conservation à travers le prisme du marché

3.1 L'expérience des banques de conservation aux États-Unis

3.2 Vers une nouvelle qualification des banques de conservation

4 Conclusion

1. Institut de Géographie et de Durabilité, Faculté des Géosciences et de l'Environnement, Université de Lausanne. 


\section{INTRODUCTION}

Depuis la Convention sur la diversité biologique, adoptée en 1992 et entrée en vigueur l'année suivante, le développement de marchés occupe une place centrale dans les politiques de conservation de la biodiversité ${ }^{2}$ L'exploitation marchande encadrée et raisonnée, avec la participation des populations locales, des autorités environnementales et d'acheteurs ou clients potentiels au pouvoir d'achat élevé, est désormais présentée comme la voie privilégiée pour assurer la gestion durable des espèces et des écosystèmes menacés ${ }^{3}$. À la faveur de la montée du libéralisme et des redéfinitions conséquentes des politiques publiques, la dégradation de la nature est interprétée comme le résultat d'un sous-investissement chronique dans la conservation, lui-même dû au défaut de droits de propriété qui affecterait les éléments de l'environnement, redéfinis comme ressources ou comme actifs naturels. Dans la mesure où personne ne peut s'assurer l'exclusivité des retours sur les investissements consentis pour la protection d'espèces ou de leurs habitats, l'incitation à réaliser de tels investissements serait faible. C'est le caractère de « bien public » de la nature qui est en cause, ainsi que le décalage entre l'apparition d'opportunités nouvelles de valorisation économique de la biodiversité et la définition du droit afférent. De même, les valeurs de la nature - et partant les coûts liés à sa destruction - ne se retrouveraient que très imparfaitement exprimées dans le système de prix, de sorte qu'elles seraient largement invisibles et échapperaient aux calculs et stratégies des acteurs économiques, d'où la sous-optimalité de leur gestion ${ }^{4}$. Les ressources biologiques seraient donc menacées du fait de leur extériorité à l'égard de la régulation marchande, cette dernière étant considérée comme solution et non plus comme problème.

Cependant, si le marché est la référence centrale de la théorie économique dominante, l'idéal vers lequel elle appelle à tendre pour assurer une allocation efficace des ressources, elle ne traite pas de la création des marchés dans ses dimensions pratiques. Différentes modalités institutionnelles ont été testées au cours des deux dernières décennies pour tenter de donner corps à la prescription d'établir des marchés pour la biodiversité. Au fil des années, les spéculations économiques ont porté successivement sur différents éléments de la nature, tour à tour considérés comme objets prioritaires des marchés en devenir. Au début des années 1990, les espoirs étaient attachés aux ressources génétiques des plantes et à la bioprospection en vue d'applications pharmaceutiques pour valoriser les forêts tropicales et autres

2. Voir C. Aubertin, F. Pinton, V. Boisvert (Eds.), Les marchés de la biodiversité, Paris, IRD Éditions, 2007.

3. On peut en trouver la parfaite illustration dans les travaux de Timothy Swanson du début des années 1990. Voir par exemple Th. Swanson, "The Economics of Extinction Revisited and Revised: A Generalised Framework for the Analysis of the Problems of Endangered Species and Biodiversity Losses », Oxford Economic Papers, New Series, vol. 46, Special Issue on environmental economics, Oct. 1994, pp. 800-821.

4. C'est l'hypothèse qui sous-tend l'exercice d'évaluation intitulé The Economics of Ecosystems and Biodiversity (TEEB) lancé à l'initiative conjointe de la Commission européenne et de l'Allemagne, lors d'un sommet des ministres de 1'Environnement du G8+5 à Potsdam en 2007, et dont l'objectif affirmé était donc de « rendre les valeurs de la nature visibles ». Voir www.teebweb.org. 
milieux riches en biodiversité. Face au faible développement de cette activité et à la complexité de mise en œuvre du cadre institutionnel adéquat, les attentes se sont en partie reportées sur les produits alimentaires et cosmétiques issus de la biodiversité. La valorisation de ces derniers passe par la segmentation des marchés et la création de niches à haute valeur ajoutée, à travers des certifications par exemple. Depuis le milieu des années 2000, ce sont les services écosystémiques qui cristallisent toute l'attention, et les dispositifs destinés à leur valorisation qui sont mis en avant dans les politiques environnementales. Un certain nombre de politiques ou d'instruments existants ont été réévalués à l'aune de leur capacité à valoriser les services écosystémiques ou ont été réorientés pour pouvoir être mobilisés dans cette perspective. C'est le cas des mécanismes de compensation, dont les banques de conservation, qui ont pour objet de compenser la destruction de l'habitat d'espèces menacées. Les banques de conservation sont ainsi présentées depuis quelques années comme de nouveaux instruments de marché au service de

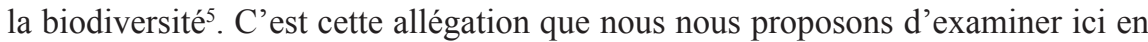
nous intéressant en particulier au statut économique des arrangements institutionnels mis en place dans ce cadre.

Si les banques de conservation sont une innovation institutionnelle relativement récente, le principe de compensation n'est en revanche pas nouveau. Il est prévu dans la législation environnementale de nombreux pays comme dernière étape de la séquence éviter/réduire/compenser. Au-delà des différences d'un pays à l'autre, le principe général en est qu'il convient d'éviter de causer des dommages aux habitats des espèces protégées dans le cas de projets d'aménagement, de faire en sorte de réduire les impacts s'ils ne peuvent être complètement évités, et enfin de compenser les dommages résiduels. Comme jusqu'à une période récente, les mesures de compensation étaient généralement réalisées en nature à proximité directe des sites affectés, elles étaient considérées plutôt comme une étape technique de la réalisation de projets d'aménagement que comme un instrument de politique environnementale à part entière. Elles ne suscitaient pas d'attention particulière de la part du monde académique, ni du reste d'un cercle plus large que les acteurs directement impliqués.

Une nouvelle modalité de compensation a émergé depuis le début des années 2000, d'abord aux États-Unis, sous le nom de « banque de conservation ». Adapté en France avec la création de la Réserve d'actifs naturels de Cossure, par la CDC biodiversité, ce nouvel instrument suscite de nombreuses prises de position, pour ou contre ce qui est présenté comme une forme de marchandisation de la biodiversité

5. Voir B. Madsen, N. Carroll, K. Moore Brands, State of Biodiversity Markets Report: Offset and Compensation Programs Worldwide, Washington, D.C., Forest Trends, 2010 ; B. Madsen, N. Carroll, D. Kandy, G. Bennett, G., Update: State of Biodiversity Markets, Washington, D.C., Forest Trends, 2011.

6. Sur un modèle similaire, on peut citer également les projets pilotes mis en place au Royaume-Uni, Defra, Biodiversity Offsetting Pilots Technical Paper: The Metric for the Biodiversity Offsetting Pilot in England, London, Defra, 2012 ; Defra, Biodiversity Offsetting in England: Green Paper, London, Defra, 2013. 
Ce dispositif est inspiré par la politique de compensation des zones humides mise en place aux États-Unis à partir des années 19807, à la suite du mot d'ordre lancé par l'Administration Bush du no net loss, autrement dit de l'équivalence écologique. L'objectif explicite de la politique de compensation est devenu de remplacer totalement les fonctionnalités des zones humides détruites, par la production d'équivalents grâce à des techniques de génie écologique. La destruction de la nature par des projets d'aménagement est ainsi rendue acceptable par la promesse que des substituts pourront lui être apportés. La réalité et la disponibilité des techniques et savoirs qui permettraient une telle (re)création de la nature ne sont pas évaluées ; c'est en tant que promesses - au sens le plus littéral du terme puisqu'il s'agit d'un engagement de campagne électorale - qu'elles sont portées dans le débat politique. Le simple fait d'avoir formulé l'objectif de no net loss permet de penser la possibilité de politiques optimales, à double dividende, assurant la conciliation entre le développement économique et la protection de l'environnement, sans sacrifice ni compromis, grâce au déploiement d'innovations techniques. Il permet de s'affranchir, au moins en rhétorique, d'un impératif à investir dans la préservation de la nature pour elle-même, l'intérêt bien compris d'entrepreneurs de la conservation y pourvoyant plus efficacement et à un coût réduit pour la collectivité.

Le dispositif appelé « banque de compensation » incarne et matérialise ce tournant libéral en matière de légitimation de la compensation. Pour assurer le no net loss, une offre de compensation sous la forme de zones humides restaurées ou créées doit être établie à l'avance. Des crédits sont émis en lien avec ces projets sous le contrôle de l'administration, en l'occurrence le Corps des ingénieurs de l'armée (U.S. Corps of Engineers). Ce sont ces projets qui sont appelés « banques de compensation », leurs promoteurs et opérateurs étant quant à eux dénommés « banquiers ». Les aménageurs qui ont une obligation de compensation peuvent se porter acquéreurs du nombre de crédits correspondant aux impacts de leur projet auprès de ces «banques ", plutôt que de réaliser les mesures appropriées euxmêmes, de se tourner vers un prestataire spécialisé qui les réaliserait en leur lieu et place, ou de s'acquitter de montants compensatoires (in-lieu fee). Les échanges de crédits se sont ainsi peu à peu développés comme modalité de mise en œuvre des obligations de compensation.

Les « banques de conservation » sont apparues aux États-Unis, avec l'ambition d'adapter ce dispositif à la protection des habitats des espèces menacées. Les premières « banques » ont été établies en Californie en $1995^{\circ}$, et ont constitué la source

7. Cette politique a été décrite et analysée dans le détail par Morgan Robertson. Voir M. Robertson, " No net Loss: Wetland Restoration and the Incomplete Capitalization of Nature », Antipode, 32(4), 2000, pp. 463-493 ; M. Robertson, « The Neoliberalization of Ecosystem Services: Wetland Mitigation Banking and Problems in Environmental Governance », Geoforum, 35(3), 2004, pp. 361-373 ; M. Robertson, « The Nature that Capital can see: Science, State, and Market in the Commodification of Ecosystem Services », Environment and Planning D: Society and Space, 2006, 24, pp. 367387.

8. D.P. Wheeler, J.M. Strock, Official Policy on Conservation Banks, The Resources Agency; California Environmental Protection Authority, April 7, 1995. 
d'inspiration des principes directeurs adoptés au niveau fédéral en 2003 par le U.S. Fish and Wildlife Service. ${ }^{9}$ 'autres programmes ont vu le jour plus ou moins sur le même modèle à partir de la fin des années 1990, en Australie, au Brésil, en Afrique du Sud et dans plusieurs pays d'Europe. Au-delà de leurs différences, ils partagent un certain nombre de prémices communes et s'appuient sur une légitimation libérale. Notre objectif ici n'est ni d'en dresser l'inventaire ni de proposer une étude de cas, mais plutôt de souligner ce que produit sur ces dispositifs le fait qu'ils soient mobilisés comme instruments de marché dans les politiques et plus encore dans la rhétorique de la conservation de la biodiversité.

Alors que la plupart des dispositifs de politiques environnementales sont aujourd'hui qualifiés d'instruments de marché, cette catégorie n'est pas aussi claire qu'il pourrait y paraître d'un point de vue analytique. La première partie de cet article sera consacrée à une présentation des notions de marché et d'instruments de marché telles qu'elles sont couramment mobilisées dans le cadre des politiques environnementales. Des critères de définition et étapes de la constitution de marchés seront dégagés, à l'aune desquels les banques de conservation de la biodiversité pourront être évaluées. À partir de l'exemple des États-Unis, le décalage entre ces instruments et les discours produits pour les légitimer ou les contester sera mis en évidence.

\section{LA NATURE DES MARCHÉS DE LA NATURE}

Les banques de conservation sont, comme on l'a évoqué, fréquemment présentées dans une veine critique comme une des dernières étapes de la marchandisation de la nature. Elles seraient le dernier avatar d'un projet sans cesse renouvelé, depuis un peu plus de deux décennies, de développer des formes de régulation marchande pour assurer la conservation de la biodiversité. La référence au marché est ainsi omniprésente dans ce contexte, mais sa portée n'est pas claire. Le terme de marché est employé dans différentes acceptions parfois confondues dans le discours : aussi bien au sens littéral que sur un mode métaphorique, pour évoquer l'idéal de marché de la théorie économique standard, mais aussi des contrats ou des transactions réelles, ou encore pour appeler au développement de secteurs d'activité particuliers. Son succès en tant que ressource rhétorique ne se dément pas, mais ce que recouvre effectivement l'injonction à étendre les marchés est plus malaisé à cerner. Derrière l'apparente limpidité ou la technicité du vocabulaire et les références à la théorie supposées parer le propos d'une certaine scientificité, des représentations et des valeurs s'affrontent.

9. U.S. Fish and Wildlife Service, Guidance for the Establishment, Use, and Operation of Conservation Banks, May 2003. 


\subsection{Le développement des marchés et des instruments de marché : quelle légitimité théorique?}

Dans la théorie économique standard, le concept de marché - de concurrence parfaite - est central. Il représente l'idéal type auquel on se réfère pour évoquer l'efficience en matière d'allocation des ressources. Le marché, dont il est question dans ce contexte, est une abstraction, définie à travers un jeu d'hypothèses ad hoc sur les comportements individuels, l'information et la nature des échanges et des objets échangés. Ces hypothèses ne sont aucunement dictées par un souci de réalisme, elles constituent des conditions mathématiques pour que la formalisation à laquelle elles donnent lieu puisse aboutir au résultat recherché : la démonstration de l'existence d'un équilibre. Paradoxalement, compte tenu de sa centralité dans l'analyse, le marché n'est pas défini de façon substantielle, mais uniquement par défaut et à travers un jeu d'oppositions : il se distingue des institutions - qui doivent être mises en œuvre, résultent de l'action collective, elle-même le fruit de préférences et de comportements individuels -, il s'oppose à l'État, incarne l'absence de contraintes, de délais, l'efficacité. Il présente une forme d'immanence et son existence n'a pas à être justifiée ni expliquée. La seule prescription politique que l'on puisse tirer d'une telle représentation est qu'il serait souhaitable de faire tendre la régulation réelle et ses institutions vers l'idéal décrit par le modèle, ce qui équivaut à une libéralisation de l'économie et à une expansion de la sphère du marché. Toutefois, la théorie n'est pas très explicite sur les modalités concrètes de mise en œuvre d'un tel programme. Sauf à considérer, à la suite d'Hayek, que le marché se développe de façon spontanée, ce qui reste une position très marginale, un cadrage institutionnel minimal est requis sitôt que l'on cesse d'imaginer le marché sous un angle purement théorique et que l'on se préoccupe de sa construction. Or l'analyse économique standard ne saurait décrire la marchandisation en tant que processus - de construction de marchandises, de définition de droits, de mise en place du cadre institutionnel permettant les transactions. Le raisonnement en statique comparative déployé ne se prête pas à l'appréhension et à la compréhension des dynamiques de changement institutionnel.

La théorie économique et sa démonstration supposée de la supériorité du marché comme mode de régulation de la biodiversité sont généralement évoquées presque pour mémoire, comme élément de légitimation théorique d'une approche libérale de la conservation. Elles sont en revanche rarement exploitées de façon systématique pour aller jusqu'à prôner une véritable mise en marché de la biodiversité $^{10}$. Le terme de marchandisation n'est d'ailleurs employé que par ses détracteurs,

10. On peut tout de même citer dans cette perspective les travaux de Geoffrey Heal. Par exemple, G. Heal, "Biodiversity as a Commodity », PaineWebber Working Paper Series in Money, Economics and Finance, Columbia Business School, Columbia University, 1999, PW-99-07 ; OCDE, Manuel pour la construction de marchés de la biodiversité : principaux enjeux, Paris, OCDE, 2005. Le projet de ce dernier ouvrage étant toutefois de développer les incitations économiques à la conservation de la biodiversité, plutôt que de la « marchandiser ». 
en association avec une dénonciation de la néolibéralisation de la nature et d'un mouvement d'enclosures du vivant qui conduirait à sa privatisation.

L'application d'un programme libéral, en matière de conservation de la biodiversité, est qualifiée par ses promoteurs non pas de création de marchés, mais d'adoption d' « instruments de marché ». Cette notion, souvent désignée par son acronyme en anglais MBI pour market-based instruments, a été initialement avancée dans le domaine environnemental par Robert Stavins, dans un rapport présenté au Congrès des États-Unis intitulé Project $88^{11}$. L'auteur y propose une classification des instruments de politique environnementale, qui fait plus ou moins référence depuis aux États-Unis. Des dispositifs très variés y sont qualifiés d'instruments de marché : taxes, subventions, redevances, marchés de droits et de permis, systèmes de certification et labels divers, réformes ou suppression des incitations perverses et mise en place d'un cadre juridique propice au développement des échanges. Ils ne présentent pourtant pas d'unité d'un point de vue théorique et ne témoignent pas d'une vision partagée des externalités environnementales. L'appellation d'instruments est même abusive pour certains des aspects des politiques passés en revue.

Jusqu'à ce que cette terminologie fasse une percée notable et s'impose notamment en relation avec la thématique des services écosystémiques ${ }^{12}$, les instruments de politique environnementale étaient plutôt classés en grandes familles selon leur inspiration théorique ${ }^{13}$. On distinguait alors les instruments fiscaux d'inspiration Pigouvienne - les outils faisant de l'État l'acteur principal de l'internalisation des externalités - et les incitations ou instruments qualifiés d'économiques, associés aux travaux de Coase - les marchés de droits et les instruments s'appuyant plus explicitement sur la régulation contractuelle ou marchande. Les premiers constituent une forme de régulation par les prix, alors qu'avec les seconds, la régulation s'effectue par les quantités - de prélèvements de ressources ou d'émissions de polluants, par exemple. Dans les travaux plus appliqués ${ }^{14}$, d'autres types de mesures étaient évoqués, faisant moins explicitement écho à la théorie économique, tel le développement de régimes de responsabilité pour dommages environnementaux. De même, la dichotomie État-marché était parfois relativisée et tempérée, pour aborder plutôt la question des autorités en charge de l'environnement sous l'angle des échelles et des lieux effectifs de décision et de contrôle. Cette grande partition

11. Ce rapport est issu d'une initiative bipartisane soutenue par les sénateurs Wirth et Heinz. Voir R.N. Stavins (Ed.), Project 88: Harnessing Market Forces to Protect Our Environment, sponsored by Senator Timothy E. Wirth, Colorado, and Senator John Heinz, Pennsylvania, 1988, Washington, D.C ; R.N. Stavins (Ed.), Project 88-Round II Incentives for Action: Designing Market-Based Environmental Strategies, sponsored by Senator Timothy E. Wirth, Colorado, and Senator John Heinz, Pennsylvania, 1991, Washington D.C.

12. Voir V. Boisvert, P. Méral, G. Froger, « MBIs for Ecosystem Services: Institutional Innovation or Renovation? », Society and Natural Resources, 2013, 26(10), pp. 1122-1136.

13. Voir, par exemple, OCDE, The Application of Economic Instruments for Environmental Protection, Paris, OCDE, 1989.

14. M. Cropper, W. Oates, « Environmental Economics: a Survey », Journal of Economic Literature, 1992, 30(2), pp. 675-740. 
et ces définitions de référence restent celles des inventaires et des évaluations des instruments de politique environnementale réalisés périodiquement par l'OCDE. Elles sont en revanche brouillées par la catégorie d'instruments de marché qui les englobe toutes indifféremment et témoigne donc plus d'une prise de position politique que d'un ancrage dans une légitimité théorique.

Cette catégorie est ainsi mal définie d'un point de vue analytique. Ce sont moins les attributs objectifs que les qualités projetées sur ces mécanismes, en l'occurrence une allocation efficace des ressources, et le fait qu'ils s'appuient très explicitement sur la régulation marchande, qui contribuent à les qualifier d'instruments de marché. Ces derniers sont ainsi couramment décrits comme exploitant les forces du marché ${ }^{15}$. Certains auteurs insistent en outre sur le fait qu'ils relèveraient de la régulation par les prix plutôt que par les quantités ${ }^{16}$. Toutefois, cette proposition ne résiste pas à l'examen : les marchés de droits, toujours identifiés comme instruments de marché, constituent un mode de régulation par les quantités. En réalité, les instruments de marché sont le plus souvent définis en creux, par la négative, en opposition à l'approche de command and control, c'est-à-dire la réglementation pure, l'édiction de normes uniformes et non négociables qui incarnerait inefficacité, lourdeur administrative et rigidité. En effet, cette dernière imposerait une contrainte identique à chaque firme, ne tiendrait aucun compte de leurs différences de coûts et de technologies ni de leur environnement concurrentiel et serait à la fois inadaptée d'un point de vue économique et peu incitative en matière environnementale.

Qualifier un instrument de politique environnementale d'instrument de marché ne dit ainsi pas grand-chose de ses caractéristiques intrinsèques. En cela, cette notion s'avère aussi peu opérationnelle que le concept de marché en économie, même si on peut en retenir que la gamme des arrangements institutionnels et des dispositifs qu'elle recouvre est vraisemblablement assez étendue, et que le rapport que ces derniers entretiennent avec le marché peut être indirect, voire se limiter à des analogies formelles. Appeler au développement d'instruments de marché est donc une prescription bien trop floue pour que l'on puisse en tirer des critères précis à l'aune desquels évaluer des processus institutionnels et pouvoir qualifier la mise en économie de la nature.

15. En anglais harnessing market forces, expression qui revient fréquemment sous la plume de Robert Stavins et en relation avec les travaux de l'OCDE. Voir notamment, R. Stavins, " Harnessing Market Forces to Protect the Environment », Environment 31 (1), 1989, pp. 5-7 et 28-35 ; OCDE, Mobiliser les marchés au service de la biodiversité. Pour une politique de conservation et d'exploitation durable, Paris, OCDE, 2003.

16. J. Hockenstein, R. Stavins, B. Whitehead, « Creating the Next Generation of Market-Based Environmental Tools », Environment 39 (4), 1997, p. 14. 


\subsection{La construction des marchandises et des arrangements marchands ${ }^{17}$}

Les termes employés pour décrire les banques de conservation - banques, banquiers, crédits, débits, etc. - témoignent de l'aspiration à construire une économie, voire une finance, de la nature et à enrôler des entrepreneurs privés dans les projets de conservation. Les banques de compensation pour les zones humides aux États-Unis sont couramment décrites comme des instruments de marché et, à ce titre, elles sont présentées comme efficientes et transparentes. Elles réduiraient les coûts de transaction en permettant une standardisation des procédures et en favorisant l'instauration de routines entre les différents acteurs de la compensation. Elles offriraient des garanties de conservation à long terme des écosystèmes, à des échelles et selon des modalités cohérentes d'un point de vue écologique. Il ne s'agit pas de leurs caractéristiques observées ni d'atouts avérés, mais des vertus habituelles attribuées au marché dans une veine libérale. Elles procéderaient à l'inverse, selon les critiques, d'un projet de marchandisation de la nature. En pratique, Morgan Robertson a montré que la mise en économie des zones humides n'était pas aussi aboutie que pouvaient le suggérer les prises de position respectives de leurs promoteurs et de leurs détracteurs, et que les banques de compensation produisaient ce qu'il a appelé une « capitalisation incomplète de la nature ${ }^{18}$. Pour les banques de conservation des habitats d'espèces menacées, dispositifs plus récents et beaucoup moins étendus, on peut s'interroger de façon plus radicale sur la qualification du processus. Peut-on légitimement parler de marchandisation? De capitalisation, fût-elle inachevée?

Comme nous l'avons vu, la théorie économique standard ne saurait apporter les outils analytiques pour appréhender l'émergence des institutions. Pour trouver les ressources théoriques requises, il convient de se tourner vers différents courants se réclamant de l'institutionnalisme - sociologique, historique, etc. - ou vers des disciplines et approches dont l'économie réelle est l'objet. Ainsi, la sociologie économique peut s'avérer particulièrement fructueuse pour comprendre les dispositifs marchands, ces derniers étant définis par Fabian Muniesa et ses collègues comme « les assemblages matériels et discursifs qui interviennent dans la construction des marchés $»^{19}$. Au-delà des différences de vocabulaire et de perspectives, ces

17. Les termes les plus couramment utilisés pour parler des marchés dans une perspective analytique (et non normative) en économie des institutions sont ceux d' « arrangements institutionnels ». La sociologie économique, à la suite de Michel Callon, emploie plutôt le terme d'agencement. Voir M. Callon, « Qu'est-ce qu'un agencement marchand? », in M. Callon et al., Sociologie des agencements marchands, Paris, Presses des Mines, 2013, pp. 325-440.

18. M. Robertson, « No net Loss: Wetland Restoration and the Incomplete Capitalization of Nature », op. cit.

19. F. Muniesa, Y. Millo, M. Callon, 2007, « An Introduction to Market Devices », Special Issue, Sociological Review Monograph Series, Market Devices, The Sociological Review, 2007, 55 (s2), pp. 1-12. 
différentes approches s'attardent sur les étapes de développement de nouvelles institutions et décrivent les opérations de cadrage qu'elles supposent.

Établir un marché implique de définir l'objet (la marchandise), les droits de propriété des protagonistes, et de mettre en place les règles et dispositifs matériels permettant l'agrégation, puis la confrontation de l'offre et de la demande afin que les transactions puissent s'opérer. Quelle que soit la forme qu'il prenne en pratique, le marché suppose en outre l'autonomie des agents économiques, dont la participation aux échanges doit être volontaire. Il implique par-dessus tout un prix, déterminé par la confrontation de l'offre et de la demande agrégées pour la marchandise et indépendant des conditions dans lesquelles s'opèrent les transactions singulières, notamment des relations - personnelles, sociales, de pouvoir - entre les acteurs concernés.

Comme cela est généralement souligné dans les travaux consacrés à la marchandisation ou la néolibéralisation de la nature ${ }^{20}$, les « marchés » liés à l'environnement se distinguent de marchés plus conventionnels dans la mesure où on a affaire à des marchandises fictives au sens de Polanyi, c'est-à-dire à des éléments qui n'ont pas été produits en vue de l'échange marchand et qui ne peuvent acquérir ce statut qu'au prix de transformations majeures. Le fait qu'elles se trouvent qualifiées ainsi témoigne d'une expansion du capitalisme et de la régulation marchande à des sphères qui en avaient été jusqu'alors exclues, ce que David Harvey ${ }^{21}$ qualifie d'« accumulation par dépossession » pour en dénoter la violence et le fait qu'elle procède de la négation de droits et de valeurs, de l'aliénation et de la spoliation, au moins autant que de la production de nouvelles représentations et de l'affirmation des statuts associés. La création de marchandises à partir de l'environnement suppose ainsi des opérations spécifiques :

- l'individuation, autrement dit la distinction d'éléments constituant la nature ${ }^{22}$, l'identification ou la construction de limites matérielles et juridiques qui permettent le démembrement des écosystèmes, leur réduction à des entités séparées, et la création de droits de propriété bien définis et échangeables sur ces ressources ou services. Notons que, dans ce contexte, ce qu'on qualifie de droits de propriété - property rights - ne renvoie pas à proprement parler ou du moins pas nécessairement à la propriété - ownership -, mais plutôt à des faisceaux de droits d'usage et d'accès - bundles of rights - relatifs aux ressources ou services liés aux écosystèmes. Cette étape est parfois qualifiée de privatisation, notamment par les géographes radicaux qui s'inscrivent dans une tradition marxiste ${ }^{23}$ ou par la nouvelle économie des institutions ou la théorie économique des droits de propriété. Le terme n'est cependant pas à prendre

20. N. Castree, "Commodifying what Nature? », Progress in Human Geography, 2003, 27(3), pp. 273-297 ; E. Gómez-Baggethun et M. Ruiz-Pérez, « Economic Valuation and the Commodification of Ecosystem Services », Progress in Physical Geography, 2011, 35(5), pp. 613-628.

21. D. Harvey, The New Imperialism, Oxford, Oxford University Press, 2003.

22. N. Castree, « Commodifying what Nature? », op. cit.

23. Ibid., p. 279. 
dans son acception littérale. Dans le premier cas, il est porté au crédit d'un discours de dénonciation de l'accaparement des communaux, du démantèlement d'institutions collectives de gestion des ressources au profit de quelques-uns et de l'expansion du capitalisme aux dépens d'économies morales. Dans l'autre, il est employé pour saluer le développement de droits de propriété individuels comme triomphe de la rationalité économique et de la raison libérale, signe de progrès et promesse d'efficacité dans la gestion des ressources. Parler de privatisation dans ce contexte ne relève donc pas du constat - et il serait erroné de le comprendre ainsi -, mais d'un parti pris normatif ;

- la réification de ces éléments, de façon à en autoriser l'aliénabilité. Ils doivent être (re)définis comme choses ou objets, physiquement et moralement séparables des sujets, lesquels sont eux-mêmes redéfinis à travers ce processus comme leurs possesseurs, et sont appelés à devenir des vendeurs dans la perspective de transactions futures ;

- l'abstraction, le fait de rendre des objets comparables, de les réduire à une dimension commune ${ }^{24}$, ce qui suppose l'établissement de conventions d'équivalence ${ }^{25}$, de classes, et un travail de nomenclature. Ce dernier requiert, selon Noel Castree $^{26}$, une double abstraction, au sens où il produit des catégories générales à partir d'objets singuliers, et où ceux-ci sont pensés indépendamment de leur localisation, de leurs appartenances et de leurs attachements. Ainsi, pour établir des banques de conservation et instaurer une commensurabilité entre nature détruite et nature recréée ou préservée, il faut dresser des typologies d'écosystèmes, selon les espèces qu'ils abritent ou l'intensité des menaces dont ils sont l'objet ${ }^{27}$. Il faut pouvoir classer et comparer des entités distinctes et considérer que celles qui appartiennent à la même classe sont substituables. Les habitats d'espèces menacées sont donc conceptuellement isolés des dynamiques écologiques singulières auxquelles ils prennent part et considérés indépendamment des attachements patrimoniaux qu'ils pourraient $\operatorname{susciter}^{28}$;

- la mesure et la quantification ${ }^{29}$, la production d'unités d'échange fongibles. La question des métriques adaptées est particulièrement complexe dans le cas de

24. D. McKenzie, « Making Things the Same: Gases, Emission Rights and the Politics of Carbon Markets », Accounting, Organizations and Society, 2009, 34, pp. 440-455.

25. Voir A. Desrosières, The Politics of Large Numbers: a History of Statistical Reasoning, Cambridge MA, Harvard University Press, 1998 ; W. Espeland, M. Stevens, « Commensuration as a Social Process », Annual Review of Sociology, 1998, 24, pp. 313-343.

26. N. Castree, « Commodifying what Nature?», op. cit., p. 281.

27. J. Salzman, J.B. Ruhl, « Currencies and the Commodification of Environmental Law », Stanford Law Review, 2000, 53, pp. 607-693.

28. Michel Callon qualifie ces opérations d'individuation, de réification et d'abstraction considérées globalement de processus de passivation. M. Callon, «Qu'est-ce qu'un agencement marchand? », op. cit.

29. Ce qui renvoie plus ou moins au néologisme de « qualcul » forgé par M. Callon, ibid. Voir aussi M. Callon et F. Muniesa, « Les marchés économiques comme dispositifs collectifs de calcul », in M. Callon et al., Sociologie des agencements marchands, op. cit., pp. 195-233. 
la biodiversité et des services écosystémiques ${ }^{30}$. Comment définir des unités de la diversité ? Quelles dimensions des écosystèmes privilégier pour pouvoir les classer et les comparer, rendre compte de leur complexité et traduire leur état de façon globale?

Une fois ces opérations effectuées, reste à identifier les ayants droit, à déterminer quels acteurs peuvent être habilités à intervenir sur le marché en qualité d'acheteurs ou de vendeurs. Il faut définir leurs droits et leurs prérogatives et en fixer les limites. Il convient de déterminer quelle autorité aura la charge de veiller au bon fonctionnement des échanges. Il faut enfin fixer un ensemble de règles et de procédures, mettre sur pied des centres de calcul assurant la mesure des valeurs échangées, et une organisation permettant la centralisation, l'agrégation et la diffusion de l'information ainsi que la réalisation matérielle des transactions.

Un premier enjeu de la qualification du statut économique des banques de conservation réside donc dans l'analyse de la mise en économie ou de l'économicisation $^{31}$ de la biodiversité qu'elles opèrent. Pour cela, on décrira la mise en œuvre et le degré d'avancement des différentes opérations mentionnées plus haut - individuation, réification, abstraction, quantification et mesure. Il conviendra d'identifier quel est l'objet de l'échange dans les banques de conservation, autrement dit ce que représentent les crédits, ce qui donne effectivement lieu à paiement et ce sur quoi porte le versement réalisé.

Ensuite, une marchandise est un bien approprié, et il est d'usage courant en économie de considérer, à la suite de Ronald Coase, que tout échange se réduit en dernière analyse à un échange de droits de propriété. Pour pouvoir évaluer ce qui se joue à cet égard dans les banques de compensation, il faut décrire les arrangements institutionnels dans leur complexité, en mettant au jour les droits et obligations des parties prenantes aux échanges de crédits, mais aussi le faisceau de relations et de transactions dans lesquelles ils s'enchâssent.

\section{LES BANQUES DE CONSERVATION À TRAVERS LE PRISME DU MARCHÉ}

De nombreux travaux retracent la brève histoire des banques de compensation aux États-Unis, depuis le développement de la compensation pour les zones humides jusqu'à leur adaptation en Californie pour la destruction d'habitats d'espèces

30. Voir J. Salzman, J.B. Ruhl, « Currencies and the Commodification of Environmental Law », op. cit.; S. Walker, A. Brower, T. Stephens, W. Lee, « Why Bartering Biodiversity Fails », Conservation Letters 2, 2009, pp. 149-157.

31. Pour reprendre les termes de K. Çalişkan et M. Callon, « Economization, part 1: Shifting Attention from the Economy towards Processes of Economization », Economy and Society, 2009, 38(3), pp. 369-398. 
protégées au milieu des années $1990^{32}$. Il existe par ailleurs une abondante littérature consacrée à l'Endangered Species Act (ESA) et à son application, particulièrement en économie du droit et en relation avec des questions de propriété ${ }^{33}$. Les expériences de différents pays en la matière ont également donné lieu à des évaluations critiques ${ }^{34}$.

Si c'est aux États-Unis que les banques de compensation ont été les plus précocement développées et les plus étudiées, les trajectoires de cet instrument dans les autres pays l'ayant adopté sont assez comparables. Jusqu'à la fin des années 1980, la compensation constituait une étape d'une procédure administrative, dont la mise en œuvre était négociée localement, au coup par coup, et soumise à de nombreuses contingences locales. Au-delà du projet particulier visé, les mesures de compensation négociées dépendaient souvent du maître d'ouvrage, de sa capacité technique à les réaliser et des moyens à sa disposition. La proportionnalité entre les dommages environnementaux anticipés des projets et les actions de restauration entreprises était envisagée de façon très fruste. Les compensations réalisées étaient décidées et réalisées au fil des projets d'aménagement, sans schéma global préalable et sans objectif explicite de rétablissement des espèces. Les représentations de la compensation des dégradations causées aux habitats, et partant la compensation elle-même, ont évolué à la suite du tournant libéral des années 1980.

Pour autant, dans la plupart des pays, le changement de perspective et l'évolution des fonctions assignées aux dispositifs de compensation ont lieu dans un cadre juridique inchangé ou du moins qui n'a pas connu de bouleversement radical. La destruction intentionnelle des habitats d'espèces menacées doit toujours revêtir un caractère exceptionnel, elle est soumise à un régime d'autorisation, dans le cadre duquel l'aménageur doit s'engager à suivre la séquence éviter/réduire/compenser.

32. M. Bauer, J. Fox, M. Bean, « Landowners Bank on Conservation: the U.S. Fish and Wildlife Service's Guidance on Conservation Banking », Environmental Law Report, 2004, 34, pp. 10717 10722 ; M. Bean, R. Kihslinger, J. Wilkinson, « Design of US Habitat Banking Systems to Support the Conservation of Wildlife Habitat and at-risk Species ", The Environmental Law Institute, Washington D.C., 2008 ; N. Carroll, J. Fox, R. Bayon, Conservation and Biodiversity Banking. a Guide to Setting up and running Biodiversity Credit Trading Systems, London, Earthscan, 2008.

33. Voir, par exemple, O.A. Houck, «The Endangered Species Act and its Implementation by the U.S. Departments of Interior and Commerce », University of Colorado Law Review, 1993, 64, pp. 277-370 ; O.A. Houck, "Why do we Protect Endangered Species, and what does that say about whether Restrictions on Private Property to Protect them Constitute "takings"? ", Iowa Law Review, 1995, 80, pp. 297-332 ; R. Innes, « Takings, Compensation and Equal Treatment for Owners of Developed and Undeveloped Property ", Journal of Law and Economics, 1997, 40, pp. 403-432 ; B. Thomson Jr., "The Endangered Species Act: A Case Study in Takings and Incentives », Stanford Law Review, 1997, 49, pp. 305-380 ; S. Polasky, H. Doremus, B. Rettig, « Endangered Species Conservation on Private Land », Contemporary Economic Policy, 1997, 15, pp. 66-76.

34. Voir, par exemple, EFTEC, IEEP, IUCN, The Use of Market-based Instruments for Biodiversity Protection The Case of Habitat Banking, Technical Report, London, 2010 ; S. Burgin, « Biobanking: an Environmental Scientist's View of the Role of Biodiversity Banking Offsets in Conservation », Biodiversity Conservation, 2008, 17, pp. 807-816 ; D. Norton, « Biodiversity Offsets: Two New Zealand Case Studies and an Assessment Framework », Environmental Management, 2009, 43, pp. 698-706 ; N.S. Koh, T. Hahn, C. Ituarte-Lima, A Comparative Analysis of Ecological Compensation Programs: The Effect of Program Design on the Social and Ecological Outcomes, Working Paper, Uppsala University, 2014. 
La compensation est la dernière étape d'une procédure administrative et le recours à l'acquisition de crédits auprès de banques de conservation n'est qu'une de ses modalités, comparativement peu développée au regard des commentaires qu'elle suscite. Jusqu'à une période récente, la compensation était le plus souvent réalisée par le maître d'ouvrage lui-même ou par un prestataire spécialisé payé par ce dernier.

Sous prétexte que les banques de conservation des espèces menacées sont inspirées des banques de compensation des zones humides, ces deux types d'instruments sont fréquemment confondus par les commentateurs, qui prêtent aux premières des caractéristiques mises en évidence pour les secondes. Les deux types de dispositifs sont ainsi parfois regroupés sous l'appellation de banques d'habitats (habitat banking) ${ }^{35}$. Ils présentent certes des analogies formelles, mais diffèrent notablement dans leurs objectifs et leur gouvernance. Ils relèvent d'administrations dont les compétences et domaines d'expertise, les cultures de l'évaluation et de l'aménagement, et les représentations de la nature sont différentes. Les banques de conservation auxquelles des aménageurs peuvent avoir recours pour satisfaire leurs obligations réglementaires sont aussi parfois confondues avec les mesures de compensation volontaire mises en œuvre par le secteur privé, le plus souvent les industries extractives, en particulier les entreprises minières ${ }^{36}$. Certaines d'entre elles communiquent beaucoup sur leurs actions en la matière. S'il s'agit d'étudier les performances écologiques des mesures compensatoires, la distinction n'est effectivement pas significative. Elle le devient en revanche pour aborder les dimensions institutionnelles et la gouvernance des dispositifs en question. Paradoxalement, au regard de l'intérêt qu'elles suscitent, les banques de conservation sont ainsi peu et mal décrites dans la littérature qui leur est consacrée.

\subsection{L'expérience des banques de conservation aux États-Unis}

Comme déjà mentionné, aux États-Unis, l'Endangered Species Act (ESA) interdit de prélever une espèce protégée, la prise (take) étant définie comme « harass, harm, pursue, shoot, wound, kill, trap, capture, or collect, or to attempt to engage in any such conduct ». En 1982, dans un contexte de recul des dépenses et des politiques publiques dédiées à l'environnement sous l'Administration Reagan, l'ESA

35. C'est, par exemple, le cas dans le rapport de M. Bean et al. de 2008 cité plus haut (n. 31).

36. Confusion probablement favorisée par le fait que les réseaux qui font la promotion de la compensation des habitats dégradés ont tendance à regrouper sous l'appellation de mesures compensatoires pour la biodiversité (biodiversity offsets) les compensations réglementaires et les compensations volontaires. Le plus actif d'entre eux est le Business and Biodiversity Offsets Programme (BBOP), émanation de Forest Trends, un consortium regroupant des ONG, des représentants d'entreprises et de gouvernements très actif en matière de promotion des instruments de marché dans le domaine environnemental. Voir htpp://www.bbop.forest-trends.org/; Business and Biodiversity Offsets Programme (BBOP), Biodiversity Offset Design Handbook, Business and Biodiversity Offsets Programme, Washington, DC, 2009. 
est amendé pour autoriser les prises accidentelles (incidental taking) par des propriétaires fonciers privés ou d'autres entités non fédérales, à condition que ces derniers adoptent des plans de conservation des habitats qui réduisent et compensent au moins partiellement les dégradations occasionnées ${ }^{37}$. Ces plans de conservation des habitats doivent être officiellement approuvés par le U.S. Fish and Wildlife Service ou le National Marine Fisheries Service selon l'espèce concernée, conformément à la Section 10(a)(1)(B) de l'ESA. À la suite de cette brèche ouverte dans la protection stricte des espèces menacées, l'État de Californie est le premier à développer des banques de conservation et une législation en encadrant le fonctionnement en 1995. Le Fish and Wildlife Service autorise à partir du milieu des années 1990 les aménageurs à acquérir des crédits auprès de banques de conservation pour remplir leurs obligations de compensation. Il faudra toutefois attendre 2003 pour qu'un cadre juridique soit adopté au niveau fédéral. Comme nous l'avons déjà évoqué, il s'agit d'un document d'orientation fédérale (Federal Guidance) relativement informel et qui laisse des marges d'interprétation et d'adaptation locales importantes. Ce document est décrit comme « une approche collaborative et incitative de la conservation des espèces menacées $»^{38}$. Il se distingue nettement en cela des réglementations sur la compensation des zones humides, qui ont un caractère contraignant $^{39}$. En pratique, un tel cadre juridique implique qu'il n'existe pas de méthode communément admise et partagée pour évaluer les impacts écologiques des banques de conservation et des projets d'aménagement.

L'objectif d'équivalence écologique, no net loss, voire celui de gain net ou d'impact positif net sont parfois mentionnés en lien avec le dispositif des banques de conservation, comme s'ils figuraient explicitement et de façon contraignante dans les documents qui encadrent la compensation dite réglementaire ${ }^{40}$. Il n'en est rien. La mise en marché des fonctions des zones humides et la compensation des espèces menacées et de leurs habitats, bien plus difficilement fongibles, soulèvent des questions différentes. La biodiversité est indissociable d'écosystèmes particuliers ; elle résulte de dynamiques évolutives localisées qui ne peuvent être aisément recréées ailleurs, ce qui rend son remplacement ou la production de substituts

37. ESA, Section 10 (a), 1982.

38. US Fish and Wildlife Service, Guidance for the Establishment, Use, and Operation of Conservation Banks, op. cit. Voir aussi M. Bauer, J. Fox, M. Bean., « Landowners Bank on Conservation: the U.S. Fish and Wildlife Service's Guidance on Conservation Banking », op. cit.

39. Department of Defense, Department of the Army, 33 CFR Parts 325 and 332 Corps of Engineers EPA 40 CFR Part 230, Compensatory Mitigation for Losses of Aquatic Resources ; Final Rule, Fed. Reg. 73(70), 10 avril 2008.

40. Business and Biodiversity Offsets Programme (BBOP), Resource Paper: No net loss and Lossgain Calculations in Biodiversity Offsets, Washington D.C., 2012 ; Business and and Biodiversity Offsets Programme (BBOP), To no net Loss and beyond: an Overview of the Business and and Biodiversity Offsets Programme (BBOP), Washington D.C., 2013 ; J.W. Bull, K.B. Suttle, A. Gordon, N. Singh, E.J. Milner Gulland, « Biodiversity Offsets in Theory and Practice », Oryx, 2013, 47(3), pp. 369-380 ; N. Doswald, M. Barcellos Harris, M. Jones, E. Pilla, I. Mulder, Biodiversity Offsets: Voluntary and Compliance Regimes. A Review of Existing Schemes, Initiatives and Guidance for Financial Institutions, UNEP-WCMC, Cambridge, UNEP FI, Genève, 2012. 
hautement improbables ${ }^{41}$. Les objectifs assignés aux politiques et aux mesures de compensation pour les habitats des espèces menacées sont de fait différents de ceux qui s'appliquent aux zones humides.

Les banques de conservation ont pour objet de contribuer à la défense des espèces protégées au niveau des États ou au niveau fédéral, et dont la destruction potentielle de l'habitat par un projet d'aménagement déclenche donc une procédure administrative. L'aménageur doit produire une étude d'impact environnemental de son projet, évaluer les dégradations à l'environnement que celui-ci pourrait occasionner et proposer des moyens de réduire ou compenser ces dommages dans un plan de conservation de l'habitat. Celui-ci est évalué par l'autorité environnementale compétente, le plus souvent le Fish and Wildlife Service, auquel revient la décision de délivrer - ou non - une autorisation et qui prescrit des mesures compensatoires, pouvant inclure une mention de la nature et du nombre de crédits dont l'aménageur pourrait se porter acquéreur.

Parallèlement, en anticipation des besoins de compensation, des propriétaires fonciers investissent dans des activités de conservation sur un site qui est appelé banque. Si la plupart d'entre eux sont privés, il arrive aussi que des organismes publics gèrent des banques, dans la mesure où seules les terres fédérales sont explicitement exclues du dispositif ${ }^{42}$. La législation reste assez évasive quant aux activités éligibles, ou du moins envisage une gamme assez étendue de possibilités : l'acquisition d'un habitat de grande qualité écologique, la restauration de l'habitat dégradé d'une ou plusieurs espèces protégées, l'extension d'aires protégées existantes, sous la forme de zones tampons les prolongeant ou de corridors facilitant les continuités écologiques. Le projet de banque doit être évalué et approuvé par les autorités environnementales.

Il convient de noter que les banques de préservation, qui n'ont pas pour objet de restaurer ni de recréer des habitats d'espèces menacées, n'entraînent pas de gain écologique. C'est la non-destruction qui est perçue dans ce contexte comme une action positive en faveur de la conservation, ouvrant le droit à l'émission de crédits. Les projets de ce type seraient privilégiés en pratique par les administrations environnementales. Ils offrent en effet de meilleures garanties de succès en termes d'impacts sur la dynamique des populations d'espèces menacées que des projets de reconstitution ou de connexion d'habitats. Une telle politique ouvre la voie à une destruction nette d'habitats et n'affiche donc pas un objectif de neutralité écologique, mais l'ambition plus floue de « compenser les effets négatifs [des projets] pour l'espèce $»^{43}$. Les projets d'aménagement et de banques de conservation sont

41. F. Quétier, S. Lavorel, «Assessing Ecological Equivalence in Biodiversity Offset Schemes: Key Issues and Solutions », Biological Conservation, 2011, 144, pp. 2991-2999.

42. Les banques de conservation peuvent être établies sur « des terres tribales, locales, privées ou d'État, où des gestionnaires maintiennent ou vont maintenir des habitats ». U.S. Fish and Wildlife Service, Guidance for the Establishment, Use, and Operation of Conservation Banks, op. cit., $\S 4$.

43. Ibid., p. 3 . 
ainsi évalués à l'aune de leur contribution - positive ou négative - à la stratégie de rétablissement d'une espèce protégée. C'est ce que traduisent les crédits, qu'il est donc difficile de relier sans ambiguïté à un niveau ou à un état de la population de ladite espèce ou de son habitat.

Les entrepreneurs de la conservation, qui choisissent d'investir dans des projets de banques, doivent monter un dossier d'agrément, qui comporte un plan de gestion pour la réserve qu'ils se proposent de créer, une description de leur aire de service, c'est-à-dire de la zone dans laquelle doivent être établis les projets d'aménagement dont la banque pourrait compenser les impacts. Ils doivent également indiquer le nombre et la nature des crédits de conservation qu'ils vont offrir, et préciser quels sont les indicateurs et critères de performance sur lesquels ils s'appuieront pour le suivi de leurs actions ${ }^{44}$. Ils doivent enfin s'engager à garantir un maintien de la banque à perpétuité. De ce fait, ils doivent céder une servitude de conservation permanente sur leur bien, et apporter la preuve qu'ils ont mis en place le plan de financement adéquat pour assurer la pérennité de leur projet. Les autorités environnementales accordent aux banques le droit d'émettre des crédits au vu de leurs résultats en matière de conservation - par exemple la création, la restauration ou le maintien d'une superficie donnée d'un type d'habitat préalablement décrit, ou encore le nombre de couples nicheurs. L'émission de crédits est donc conditionnée par le succès du plan de gestion.

Chaque espèce ou type d'habitat donne lieu à un type de crédit distinct et, pour chaque type de crédit, différentes méthodes d'évaluation peuvent être mises en œuvre. D'après un des opérateurs historiques de la compensation aux États-Unis, on peut identifier quatre types principaux de métriques applicables à l'estimation des habitats se déclinant en plus de 40 méthodes $^{45}$. Il n'existe ainsi pas d'unité communément acceptée sur laquelle pourraient se fonder les comparaisons des habitats détruits et des habitats préservés, restaurés ou recréés. L'absence de fongibilité des crédits traduit un travail d'abstraction inachevé ; il n'y a pas de typologies qui permettraient d'établir des classes d'équivalence entre des habitats. Ceux-ci sont fondamentalement situés, inscrits dans une trajectoire évolutive, et représentent des valeurs environnementales, sociales et culturelles dont il est impossible de les détacher ${ }^{46}$. Chaque transaction est ainsi pratiquement unique et profondément ancrée dans un contexte particulier. Les arrangements institutionnels mis en place naissent de dynamiques locales et font l'objet de négociations et d'appréciations

44. Voir M. Bauer, J. Fox, M. Bean, « Landowners Bank on Conservation: the U.S. Fish and Wildlife Service's Guidance on Conservation Banking », op. cit.

45. Willamette Partnership, Measuring up: Synchronizing Biodiversity Measurement Systems for Markets and other Incentive Programs. A Report funded by the U.S. Department of Agriculture, Office of Environmental Markets, 2011.

46. Comme le notent S. Brownlie et M. Botha, « l'utilisation d'unités frustes, de substituts approximatifs de la biodiversité et de métriques simplistes dans le cadre de la compensation ne permettent pas de rendre compte des valeurs environnementales et du bien-être social dans le temps et l'espace ». S. Brownlie, M. Botha, "Biodiversity Offsets: Adding to the Conservation Estate, or "no net Loss"? », Impact Assessment and Project Appraisal, 2009, 27 (5), p. 227. 
subjectives du sérieux et du professionnalisme des opérateurs en présence ou de leur capacité à tenir leurs engagements. L'issue des transactions est dépendante des relations établies entre les acteurs, elle ne peut être totalement objectivée et considérée comme liée aux seules caractéristiques intrinsèques de l'objet de l'échange, qu'il est d'ailleurs difficile de définir exactement. En effet, le nombre de crédits attribuables à un projet de banque et le nombre de crédits à se procurer pour compenser les effets d'un projet d'aménagement font l'objet d'évaluations distinctes, réalisées à des moments différents, avec des techniques potentiellement différentes, éventuellement en appliquant des indicateurs et ratios différents. Il est improbable que les crédits demandés et les crédits offerts soient de même nature ou traduisent une réalité similaire en termes d'impacts environnementaux ${ }^{47}$. Ce ne sont pas non plus des «droits de propriété » qui sont transférés à travers la vente de crédits. En effet, l'obligation de compenser est personnelle et la responsabilité formelle de l'exécution des mesures compensatoires revient à l'aménageur, même après l'acquisition de crédits. Cette dernière confère tout au plus de la flexibilité dans l'exécution d'obligations réglementaires. L'échange de crédits est la manifestation tangible du compromis atteint entre d'une part la banque de conservation et l'autorité environnementale, d'autre part cette dernière et l'aménageur, plus que le reflet d'un accord direct entre banque et aménageur. Il traduit d'une part la contractualisation partielle des politiques de conservation des espèces protégées, d'autre part l'assouplissement du régime de protection stricte de ces espèces et de leurs habitats. Il ne saurait être qualifié d'échange d'équivalents.

Le rôle de l'autorité environnementale ne se limite pas à une médiation entre offre et demande ni à une facilitation des transactions. Son intervention est décisive dans la définition même de l'offre et de la demande de crédits. Jessica Fox et Anamaria Nino-Murcia rapportent d'ailleurs que la plupart des « banquiers », qu'elles ont interrogés lors d'une enquête sur les banques de conservation aux États-Unis, se plaignaient de la charge administrative liée à leur activité et des nombreuses démarches qu'ils avaient à accomplir ${ }^{48}$, ce qui est peu conforme à l'image de libéralisation liée à l'émergence de ce secteur. De même, le développement des banques de conservation est parfois présenté comme une entreprise de privatisation de la conservation de la nature, un désengagement financier des autorités publiques en charge de l'environnement. Même si cela est comparativement peu fréquent, il peut arriver, comme nous l'avons déjà relevé, que les opérateurs des banques soient des acteurs publics ou parapublics, ce qui est peu souligné dans un discours de promotion d'une gouvernance néolibérale des ressources reposant sur l'initiative privée. Dans tous les pays qui se sont dotés de systèmes de ce type, le fonctionnement des banques de compensation est très étroitement dépendant

47. On peut même être assuré du contraire quand ils sont émis par une banque de préservation, et que la contrepartie proposée pour la destruction d'habitat est simplement le maintien d'habitats existants.

48. J. Fox, A. Nino-Murcia, «Status of Species Conservation Banking in the United States », Conservation Biology, 2005, 19, pp. 996-1007. 
d'un régime d'autorisation administrative, il s'appuie sur des faisceaux de contrats avec de nombreuses parties prenantes et ne saurait être ramené à la transaction par laquelle un développeur achète les crédits dont il a besoin pour compenser les dommages occasionnés par son projet.

Enfin, en dépit de l'impression qui se dégage de la littérature quant à l'explosion du phénomène des banques de conservation, son ampleur reste limitée, même aux États-Unis ${ }^{49}$. Il n'y a de banques que dans un nombre limité d'États et pour un nombre limité d'espèces protégées. Le nombre d'opérateurs de banques est comparativement bien moins élevé que dans le secteur de la compensation des zones humides, au point que certaines banques se trouvent dans des situations de monopole local. Chaque banque ne peut émettre qu'un nombre limité de crédits et une fois l'ensemble de ses crédits vendus, elle n'a plus la possibilité d'en émettre de nouveaux. Les banquiers se consacrent alors exclusivement à la gestion de leur réserve naturelle. Les crédits ne peuvent être ni thésaurisés ni revendus, ils ne peuvent faire l'objet que d'une transaction unique dans un sens toujours identique, de la banque vers l'aménageur, ce qui conforte l'idée selon laquelle ils ne permettent que de rendre plus flexible un marchandage ou un troc ${ }^{50}$, mais en aucun cas ne constituent une unité de valeur de la biodiversité. Le volume de transactions est limité puisque le niveau de la demande est complètement déterminé par les décisions de l'administration d'autoriser, à titre en principe exceptionnel, des projets qui détruisent des habitats d'espèces protégées.

On observe donc avec les échanges de crédits une multiplication de transactions uniques en lien avec la biodiversité, mais dont l'objet n'est pas à proprement parler un élément de la biodiversité et varie beaucoup en fonction des situations. Les actifs environnementaux sur lesquels s'appuient les métriques sont très différents d'un cas à l'autre. Il est donc malaisé de parler des banques en général, tant chaque banque est singulière ${ }^{51}$. La centralisation, et surtout la mise à disposition du public de l'information sur les banques à travers le Regulatory In Lieu Fee and Bank Information Tracking System (RIBITS), est beaucoup moins avancée que pour les dispositifs de compensation des zones humides. Il apparaît difficile au vu de ce contexte de parler de «marché de la biodiversité » ou de marchandisation de la nature. On observe toutefois une volonté de standardiser et de normaliser les techniques d'évaluation, de centraliser l'information sur les crédits pour que le système d'échange présente des caractéristiques de marché. Les métiers de la compensation sont en cours de réglementation à travers une organisation des experts,

49. D'après le site Internet speciesbanking qui recense les expériences de banques de conservation, il y aurait actuellement 135 banques aux Etats-Unis dont 112 en Californie, 5 en Floride, 4 au Texas et dans l'État de Washington, 3 en Oregon et en Utah, 2 en Arizona, http://www.speciesbanking.com.

50. Voir S. Walker, A. Brower, T. Stephens, W. Lee, «Why Bartering Biodiversity Fails », op. cit., p. 151 et J. Salzman, J.B. Ruhl, «Currencies and the Commodification of Environmental Law », op. cit., p. 614.

51. Ce qui est du reste relevé dans le texte d'encadrement fédéral : «In general, no two conservation banks will be used or developed in an identical fashion ». U.S. Fish and Wildlife Service, Guidance for the Establishment, Use, and Operation of Conservation Banks, op. cit., p. 1. 
consultants et courtiers divers spécialisés dans la compensation dans plusieurs pays - création d'organisations professionnelles, projet de développement de procédures d'agrément et de certification des opérateurs. Dans les présentations des banques de conservation par le Fish and Wildlife Service, il est parfois évoqué qu'elles pourraient également proposer leurs crédits dans un cadre de compensation volontaire et développer des actions de conservation visant les espèces menacées non listées. Ces possibilités d'évolution ne semblent pas en passe de se concrétiser dans l'immédiat. Elles traduisent néanmoins la conscience qu'ont les promoteurs du système que, en l'état, il est assez éloigné de l'idéal marchand réputé efficace. Les systèmes de compensation de la biodiversité mis en place relativement récemment l'ont d'ailleurs été explicitement avec des objectifs marchands. C'est ainsi le cas du système de BioBanking, établi en Nouvelle-Galles du Sud en Australie, en 2006, et dans lequel il est possible de thésauriser les crédits, mais vraisemblablement avec un succès limité à ce jour tant d'un point de vue économique que sous un angle écologique ${ }^{52}$.

Transformer en instruments de marché des outils de politique environnementale dont ce n'était pas la vocation première devient une fin en soi. Des ressources sont dédiées au développement des caractéristiques volontaires et contractuelles des banques de compensation, à la participation d'opérateurs privés - au besoin impulsée par des incitations économiques et subventions directes -, à l'émergence de courtiers, de spécialistes, d'experts qui se chargent des différentes opérations, préludes à la mise en marché plutôt qu'à la résolution directe des problèmes qu'ils visent.

\subsection{Vers une nouvelle qualification des banques de conservation}

À l'instar des banques de compensation pour les zones humides, les banques de conservation de la biodiversité sont couramment présentées comme des instruments de marché ou, sur un mode plus polémique, comme des projets de marchandisation. Une telle qualification n'est pas neutre. Elle concourt à occulter le cadre juridique dans lequel elles s'inscrivent et à ne considérer qu'un type de transactions et d'acteurs et qu'une étape d'un processus de plus long terme, au détriment du complexe plus vaste de normes, d'accords et de conventions à travers lequel les politiques de conservation s'appliquent. Elle néglige et masque certaines dimensions résolument non marchandes du système. Elle conduit enfin à postuler une efficacité de ces dispositifs sur le plan écologique qui n'est pas démontrée. En qualifiant les banques de conservation d'instruments innovants et en fort développement, on les isole par ailleurs, au moins d'un point de vue discursif, des dispositions qui les ont précédées et on exagère la rupture qu'elles auraient induite. L'assimilation fautive des banques de conservation des espèces listées d'une part avec les banques de compensation des zones humides et, d'autre part, avec les opérations de compensation volontaire

52. Voir S. Burgin, « Biobanking: an Environmental Scientist's View of the Role of Biodiversity Banking Offsets in Conservation », op. cit. 
n'est pas anodine. Elle procède d'une tentative d'affirmer le caractère marchand des banques de conservation par analogie avec ces dispositifs plutôt qu'à partir de la description de leurs caractéristiques réelles. Les banques de conservation sont ainsi observées et décrites à travers un prisme économique qui ne met en avant que les aspects susceptibles de s'accorder au discours libéral qui les a portées ${ }^{53}$. Leur analyse critique dans le champ des sciences sociales et politiques ne porte que sur la place à accorder à la régulation marchande dans le gouvernement de la nature, la description des instruments en eux-mêmes dans leur complexité étant comparativement négligée. Les discours de justification et de dénonciation du caractère marchand des banques de compensation se renforcent finalement pour contribuer à affirmer ce dernier comme une évidence. Il ne devient plus possible de traiter de cet instrument autrement que sous cet angle.

Alors même que la justification de la compensation tient à des dégradations initiales causées à l'environnement, les banques de compensation tendent à être présentées comme des outils de conservation de la biodiversité. C'est ainsi l'aspect positif de l'opération de compensation qui est le plus souvent mis en avant : l'espace restauré, ou aménagé pour assurer certains services écosystémiques, tandis que la destruction qui est son corollaire est relativement peu évoquée ${ }^{54}$. Les opérations dites de conservation de l'environnement, présentées comme vertueuses et efficaces, peuvent pourtant au mieux compenser la dégradation de la biodiversité engendrée par d'autres projets et résulter en un jeu à somme nulle. La focalisation sur le caractère marchand ou non des banques et la nature des marchés qu'elles permettraient d'instituer détourne dans une certaine mesure de ce qui affecte le plus directement la biodiversité : la banalisation des dérogations à la protection stricte des espèces listées et de leurs habitats. Des milieux peuvent être détruits contre la promesse que des milieux comparables déjà protégés le resteront à perpétuité.

S'il fallait rapprocher les banques de conservation d'instruments de politique existants, c'est probablement avec les droits de développement transférables qu'elles présentent le plus de similitudes ${ }^{55}$. Ces droits initialement utilisés pour protéger l'unité architecturale des centres-ville aux États-Unis ont été adaptés à la

53. Il en résulte ce que Ulrich Brand et Alice Vadrot appellent des sélectivités épistémiques, à savoir une appréhension tronquée de l'objet, la sélection de ses seuls aspects qui vont dans le sens d'un discours dominant, et qui deviennent surdéterminants tant pour l'analyse que dans la production de politiques. U. Brand, A. Vadrot, « Epistemic Selectivities and the Valorisation of Nature: the Cases of the Nagoya Protocol and the Intergovernmental Science-Policy Platform for Biodiversity (IPBES) », Law, Environment And Development Journal, 2013, 9(2), pp. 202-220.

54. La communication de la CDC Biodiversité sur l'opération Cossure, la constitution d'une réserve d'actifs naturels en France dans la plaine de la Crau, est emblématique à cet égard. Ce sont les actions de réhabilitation du site, de renaturation et en particulier de reconstitution de la végétation qui sont mises en avant et dont les succès sont soulignés, bien plus que le fait qu'elles sont offertes comme contrepartie de destructions ailleurs. Ce dernier aspect n'est évidemment pas occulté, mais présenté de telle façon qu'il n'est probablement pas compris par un public qui ne serait pas familier du sujet. Voir http://www.cdc-biodiversite.fr.

55. OCDE, Handbook of Incentive Measures for Biodiversity Design and Implementation, Paris, OCDE, 1999 ; OCDE, Recommendations of the Council on the Use of Economic Instruments in Promoting the Conservation and Sustainable Use of Biodiversity, Paris, OCDE, 2004. 
protection de l'environnement. Il s'agit d'offrir aux propriétaires de biens situés dans une zone à protéger, qualifiée de district émetteur, l'opportunité d'aménager ou de vendre leur droit à aménager des parcelles situées dans des zones dédiées à l'artificialisation, qualifiées de districts récepteurs. Ces droits sont une forme de compensation offerte aux propriétaires qui ne peuvent complètement exercer leurs droits sur les biens qu'ils possèdent en raison de réglementations contraignantes. Ils sont mobilisés notamment pour éviter la construction d'immeubles excédant une certaine hauteur dans des quartiers historiques. La mise en œuvre d'un tel système nécessite préalablement une partition du territoire entre districts émetteurs et districts récepteurs et la mise en place du cadre juridique permettant la vente des droits de développement transférables. Appliqué à des écosystèmes sensibles, ce dispositif se traduit par des restrictions d'activités dans des zones dédiées à la conservation, mises en œuvre à travers des servitudes ou des plans de gestion ${ }^{56}$.

Les banques de conservation sont dans une certaine mesure l'analogue de districts émetteurs, les aires de services pouvant quant à elles apparaître comme des districts receveurs, à la différence près, au demeurant non négligeable, qu'il ne suffit pas pour des entrepreneurs d'avoir des projets localisés dans ces zones pour pouvoir bénéficier de la possibilité d'acquérir des crédits. Il leur faut négocier en amont avec l'administration environnementale en vue de l'obtention d'un permis, sans garantie d'une issue favorable. Une autre différence majeure réside dans le fait que l'émission de crédits est la raison d'être d'une banque de compensation et non pas l'effet non intentionnel de restrictions foncières. Par ailleurs, les droits de développement transférables peuvent généralement être thésaurisés ou revendus à des tiers, ce qui n'est pas le cas des crédits liés à des espèces menacées ou à leur habitat, du moins aux États-Unis.

Néanmoins, ce parallèle permet de souligner que plus qu'une entreprise de marchandisation de la biodiversité, la politique de compensation relève d'une spécialisation et d'une ségrégation des espaces, qualifiée en anglais de land sparing ${ }^{57}$. L'artificialisation et le développement d'une partie du territoire sont jugés acceptables pour autant qu'ils s'accompagnent de la constitution ou de la sécurisation de réserves dédiées à la conservation. Il n'est pas démontré que ce soit la solution la plus adaptée en matière de protection de la nature ${ }^{58}$, d'autant qu'avec le

56. OCDE, ibid., 2004. La proposition d'adapter cet instrument à la conservation de la biodiversité a été avancée par T. Panayotou au début des années 1990. T. Panayotou, « Conservation of Biodiversity and Economic Development: the Concept of Transferable Development Rights ", Environmental and Resource Economics, 1994, 4, pp. 91-110.

57. B. Phalan, M. Onial, A. Balmford, R.E. Green, « Reconciling Food Production and Biodiversity Conservation: Land Sharing and Land Sparing Compared », Science, 2011, 333(6047), pp. 1289-1291.

58. R.S. DeFries, J.A. Foley, G.P. Asner, « Land-Use Choices: Balancing Human Needs and Ecosystem Function », Frontiers in Ecology and the Environment, 2004, 2(5), pp. 249-257 ; J. Fischer, P. Batáry, K.S. Bawa, L. Brussaard, M.J. Chappell, Y. Clough, G.C. Daily, J. Dorrough, T. Hartel, L.E. Jackson, A.M. Klein, C. Kremen, T. Kuemmerle, D.B. Lindenmayer, H.A. Mooney, I. Perfecto, S.M. Philpott, T. Tscharntke, J. Vandermeer, T.C. Wanger, H. von Wehrden, « Conservation: Limits of Land Sparing ", Science, 2011, 334(6056), p. 593. 
changement climatique, les aires de répartition des espèces peuvent se déplacer. En outre, la diffusion d'un tel modèle d'aménagement dans des territoires européens densément peuplés et artificialisés, où les réserves foncières sont limitées, n'est pas sans poser problème. Il est probable qu'elle s'oriente davantage vers la constitution de banques de préservation - autrement dit le maintien d'habitats déjà préservés - que vers la restauration ${ }^{59}$, ce qui peut faire craindre un recul global des habitats menacés. L'importance des réseaux écologiques, de la connectivité, des mosaïques de paysages en termes de conservation est largement débattue en écologie et une approche plus intégrée - combinant aménagement urbain ou artificialisation agricole et conservation sur le même espace et qualifiée de land sharing - est considérée comme alternative et discutée en opposition à la spécialisation spatiale. Il n'existe pas de stratégie d'aménagement qui ait démontré sa supériorité, quels que soient les objectifs de conservation et les espèces concernées. On ne saurait par conséquent légitimer d'un point de vue strictement scientifique le choix d'une concentration des espaces dédiés à la conservation. En revanche, une telle organisation des mesures compensatoires favorise leur suivi.

L'économicisation des espèces menacées et des habitats protégés dans le cadre des banques de conservation n'est ainsi pas forcément le prélude à leur marchandisation à proprement parler. Le processus de mise en nombre, d'établissement de typologies et de conventions d'équivalence est bien engagé, mais il pourrait être dicté avant tout par des impératifs de gouvernance et de contrôle. Le développement des banques de conservation des espèces menacées sur le modèle de la compensation des zones humides permettrait avant tout de remédier à l'éparpillement des actions de compensation organisées par les aménageurs eux-mêmes avec plus ou moins de succès et de professionnalisme ${ }^{60}$. Il s'agirait surtout de rationalisation, dictée par une justification managériale. La spécialisation des opérateurs et leur professionnalisation favorisent l'apparition de routines, le développement de relations de confiance et la réduction des coûts de mise en œuvre des politiques. La concentration des opérations de compensation les rend plus faciles à évaluer et à contrôler pour les pouvoirs publics. Elle aurait en outre une justification environnementale puisqu'elle permettrait une planification territoriale de la compensation et l'établissement de schémas de cohérence écologique. Le Fish and Wildife Service considère que l'argument majeur en faveur des banques est la capacité qu'elles lui confèrent à maîtriser - au moins en partie - la localisation des mesures compensatoires. Les autorités environnementales évaluent de façon indépendante, d'une part, les dommages que pourraient causer des projets d'aménagement et, d'autre part, le potentiel en termes de rétablissement des espèces menacées des banques. Ces deux transactions bien distinctes et le pouvoir qu'elles permettent d'exercer

59. M. Hannis, S. Sullivan, Offsetting Nature? Habitat Banking and Biodiversity Offsets in the English Land Use Planning System, Greenhouse, UK, 2012.

60. U.S. Fish and Wildlife Service, Guidance for the Establishment, Use, and Operation of Conservation Banks, op. cit., p. 1. 
sont occultés par la mise en avant de l'échange de crédits comme négociation directe entre aménageur et banquier. Le système des banques de conservation renforce finalement la centralité des autorités environnementales plutôt qu'il ne les marginalise ${ }^{61}$. On peut ainsi considérer qu'il relève des technologies de pouvoir ${ }^{62}$, et constitue avant tout un levier de l'action publique en matière de conservation.

L'impact de la mise en nombre de la nature est ainsi difficile à déterminer de manière catégorique. Elle est construite comme objet de gestion, mais pour autant, en dépit des projections et des discours tenus sur les banques de conservation, le degré d'avancement de sa mise en économie ne permet pas de présumer du type de régulation qui va s'imposer.

\section{CONCLUSION}

La qualification des banques de compensation comme instruments de marché révèle moins leurs caractéristiques objectives que les aspirations de leurs promoteurs et les craintes de leurs détracteurs. Les échanges de crédits, dans des conditions singulières pour chaque transaction, peuvent être perçus comme les prémices d'un marché, qui pourrait se développer avec une meilleure centralisation de l'information, une homogénéisation des crédits et une standardisation des procédures d'évaluation des dommages environnementaux et des techniques de restauration. La professionnalisation des acteurs et l'instauration de certifications dans ce sens sont également perçues comme pouvant donner des garanties précieuses. Pour autant, comme on l'a montré, la situation actuelle est très éloignée d'un idéal marchand.

L'encadrement juridique et administratif de la compensation est très présent et une bonne partie des interventions des acteurs s'inscrit dans le cadre d'obligations ou de prérogatives légales, rendant illusoire l'idée même d'une liberté contractuelle.

Certes les banques de compensation sont, au moins pour la plupart d'entre elles, des entreprises privées, dans lesquelles des investisseurs choisissent de s'engager librement. L'expansion possible de leur activité est toutefois contenue par la loi. La demande de crédits est conditionnée et plafonnée par les autorisations accordées par l'administration environnementale de déroger aux mesures de préservation des espèces menacées en portant atteinte à leur habitat. Le développement des banques est en outre limité en pratique par les possibilités d'accéder au foncier afin d'y établir des réserves à perpétuité.

61. Même s'ils ne formulent pas le constat en ces termes et ont réalisé leurs travaux empiriques sur les mécanismes de compensation des zones humides, c'est ce qui conduit Anne-Charlotte Vaissière et Harold Levrel à qualifier ces dispositifs d'hybrides entre la hiérarchie et le marché. A.-C. Vaissière, H. Levrel, « Biodiversity Offsets Markets: What are they really? An Empirical Approach to Wetland Mitigation Banking », Ecological Economics 2015, 110, pp. 81-88.

62. Pour reprendre l'expression de M. Foucault, "L'éthique de soi comme pratique de liberté », in Dits et écrits, t. IV (1980-1988), Paris, Gallimard, pp. 728-729. 
Parler de marché à propos des banques de biodiversité supposerait en outre qu'il y ait une marchandise, que l'objet de l'échange soit clairement identifié et soit effectivement transféré du maître d'ouvrage à l'opérateur de la banque par la transaction. Comme nous l'avons montré, ces deux points posent problème. La représentation des banques de conservation comme émettant des "droits à tuer » des spécimens d'espèces menacées ou plus exactement des « droits à détruire » leur habitat est abusive. L'obtention du permis de réaliser des « prises accidentelles » est préalable à l'acquisition éventuelle de crédits, même si cette dernière possibilité joue dans la négociation. Ensuite, il n'y a pas à proprement parler de transfert, au sens où l'obligation de compenser a un caractère personnel. Elle relève de la responsabilité de l'aménageur et n'est pas transférée à la banque de compensation. Il s'agit donc tout au plus d'un mécanisme de flexibilité.

Plus qu'une marchandisation des politiques de conservation, ce qui s'opère est une mise en visibilité d'une de leurs étapes, celle de l'échange de crédits, autour de laquelle se sont cristallisés les débats et les écrits au cours de la dernière décennie, au détriment des étapes antérieures des procédures, qui restent quant à elles relativement inchangées, et de défis en matière de restauration écologique qui restent toujours à relever. Les décisions qui affectent réellement les politiques de conservation interviennent probablement en marge de l'échange de crédits à proprement parler. Elles sont liées à la (re)définition des listes d'espèces et d'habitats à préserver, aux négociations sur les échelles spatiales auxquelles la compensation peut s'opérer, aux opérations de sécurisation foncière et financière des banques, aux différents documents de planification réalisés par les protagonistes, ou encore aux régimes d'autorisation.

La complexité des procédures prescrites par l'administration et les exigences à la fois scientifiques, managériales, juridiques et financières auxquelles il faut satisfaire entraînent l'essor d'un secteur d'activité spécialisé dans l'accompagnement et la facilitation de la compensation. Une nouvelle classe d'entrepreneurs de la conservation apparaît également. Si on peut parler de développement du « marché de la compensation », c'est ainsi en tant que secteur d'activité.

Néanmoins, l'emprise de l'imaginaire marchand produit des effets. Sans qu'il y ait de renversement de la séquence éviter/réduire/compenser, la compensation à travers l'acquisition de crédits se développe, et peut faire craindre que les dégradations causées à l'environnement ne se banalisent. L'idéal du no net loss, importé des univers de la compensation des zones humides et des projets de compensation volontaire menés par de grandes entreprises, change les représentations et les justifications de la compensation, d'une perspective de réparation, de restauration et de remédiation vers une perspective de production de la nature. La mise en place d'une offre de compensation indépendante des dommages causés à l'environnement peut entraîner littéralement la production d'une « seconde nature ${ }^{63}$,

63. Selon l'expression de W. Cronon, Nature's Metropolis. Chicago and the Great West, New YorkLondon, W.W. Norton \& Company, 1991. 
artificielle, façonnée par le génie écologique et conforme à un idéal en matière de conservation, de recréation d'habitats et de rétablissement des espèces. Il y a quelque chose de profondément contradictoire à vouloir produire de l'environnement « naturel » tout en décidant dans le même temps de le figer à perpétuité, pour qu'il reste conforme à la description qui en a été faite au moment de l'émission des crédits. Au-delà des modalités de la compensation, le simple fait de penser une politique de conservation en ces termes l'ancre dans un univers d'équivalence et d'aliénabilité généralisées qui peut la conditionner de façon déterminante.

\section{SUMMARY: ECOLOGICAL COMPENSATION: MARKET OR BARGAINING?}

During the last decade, conservation banks have emerged, at least in environmental discourse, as new market instruments for promoting biodiversity conservation. Compensation has already been provided for in environmental law in many countries since the 1970s as the last step of the mitigation hierarchy, but biodiversity compensatory mitigation devices have been reshaped as market-based instruments (MBIs). The promarket narrative featuring biodiversity banking systems as market-like arrangements and their denunciation as instances of the commodification of nature tend to obscure their actual characteristics and to produce and convey a biased and selective representation of these institutional devices. The purpose of this paper is to describe conservation banks, adopting an explicitly analytical stance on these complex institutional arrangements and their economic status.

While most environmental policy instruments are labelled as MBIs nowadays, this category is ill defined from an analytical point of view. Describing an environmental policy instrument as market based reveals the cost-efficiency expectations attached to its adoption rather than its objective characteristics. It is an ideological rather than a theoretical statement. The concept of MBI therefore has a limited heuristic value and cannot lead to the definition of criteria to assess real-life institutional arrangements. Building on categories and concepts developed in economic sociology and radical geography, several steps of the economization process at stake in conservation banking devices can be identified. Biodiversity can be turned into a set of tradable entities through a sequence of operations: individuation, reification, abstraction, measurement, and quantification. The assessment of biodiversity banking can be conducted based on the latter, and on the example of the US experience. In the United States, conservation banks are established, used, and operated within the boundaries of the Endangered Species Act. Conservation banks are permanently protected lands devoted to the conservation of one or several listed, endangered, or threatened species. Eligible conservation activities are diverse; they may include the acquisition of high-quality habitat, the restoration of a degraded habitat, buffering of protected areas, or connecting separated habitats. They must 
be established on nonfederal lands but might be managed by, for instance, local authorities. The bankers must set up a "conservation banking agreement" indicating a management plan for the bank property, a description of the service area, the number and kind of conservation credits within the bank, and the performance standards that it should meet. They must in addition convey a permanent conservation easement over the bank property and provide assurance of long-term funding for the perpetual management of the property. The location of the bank and its management plan are subject to the approval of the environmental authority, and credits are awarded based on conservation outcomes.

On the demand side, if a land development project may result in an "incidental taking" of a protected species, an administrative procedure is launched. The land developer must provide an ecological impact assessment of the project, estimate the potential biodiversity loss and habitat destruction that might be induced by the project, and propose measures to mitigate these damages in a Habitat Conservation Plan. This plan is assessed by the environmental authority-most of the time the US Fish and Wildlife Service-that delivers a permit and prescribes compensatory measures, possibly including the specification and nature of the credits that the developer should buy.

Biodiversity banking is therefore organized through bundles of contracts, agreements, and binding planning documents, involving many stakeholders over the long term. There is a distinct type of credit for each species and each habitat type, and for each of them different ecological assessment methods can be used. The credits are not fungible, and each transaction is unique and embedded in a particular context from which it cannot be abstracted. The purchase of credits cannot be depicted as a voluntary transaction and it is not an exchange of property rights either, since it does not involve any transfer of formal rights. Indeed, offsetting requirements are a matter of personal obligation that cannot be transferred.

In fact, the economization of endangered species and their habitats could be interpreted as part of a land-sparing strategy, guided by managerial rather than commodification objectives. Far from the market narrative supporting its development, conservation banking is perceived by environmental authorities as instrumental in rationalizing and reinforcing their control over conservation activities and their location. Rather than market-based instruments, conservation banks could be defined as technologies of the market-that is, technologies of power in the Foucauldian sense.

Mots clés : marchandisation, instruments de marché, banques de conservation, biodiversité

Keywords: commodification, market-based instruments, conservation banking, biodiversity 